\title{
X-ray Tomography and Finite Elements Simulations of Rock Mechanics
}

\author{
D. Müter ${ }^{1}$, H. O. Sørensen ${ }^{1}$, D. Jha ${ }^{1}$, K. N. Dalby ${ }^{1}$ and S. L. S. Stipp ${ }^{1}$ \\ ${ }^{1}$ University of Copenhagen, Nano-Science Center, Copenhagen, Denmark
}

Chalk cliffs in many countries, such as around the North Sea in Europe, are places of natural beauty but also under the constant threat of erosion. Collapse of chalk cliffs, which are often poorly consolidated (Figure 1), have cost lives and destroyed property and cultural heritage with a concerning frequency. Predicting collapse, however, is difficult and has had little success in the past. To assess the stability of these cliffs it is necessary to know about the mechanical properties of the rock. On the macroscale, common mechanical testing, as performed in engineering, can help to provide estimates but ultimately, it is the morphology of the nanoscale pore system in chalk, or any poorly consolidated material, that determines its macroscopic mechanical properties.

The particles and pore space, thus the controlling features of chalk structure, are on the nanoscale which restricts the use of laboratory based analysis because of limitations in resolution. A few synchrotron radiation facilities worldwide have dedicated high resolution tomography beamlines with a voxel size as small as $25 \mathrm{~nm}$ (e.g. at beamline ID 22 at the ESRF [1]). Even with this state-of-the-art equipment, resolving the pore structure in chalk is a challenging task and requires a fair amount of image processing. First, ring artefacts and other noise must be removed from the tomography data. To segment the data into material and pore phase, we developed our own technique that uses a three dimensional Unsharp Mask filter and conjunction with median filtering to preprocess the data [2]. The segmentation itself is then performed using traditional Otsu thresholding.

From the segmented data, primary parameters can easily be calculated. The porosity is given by volume of pore space voxels (= volume pixels) in relation to the total number of voxels whereas the surface area can be determined from the total number of voxel faces at the interface between the material and pore phase. To assess the mechanical properties, we choose a subvolume of the segmented data and produce a mesh that is optimized for the local curvature in the sample, i.e. more and smaller elements around fractures and pores [3]. Subsequently, the meshed volume is imported into finite elements software [4]. This enables us to directly simulate mechanical deformation under a range of loading conditions, i.e. compression and tension along different axes. By applying a small load onto the sample and calculating the deformation (Figure 2), we are able to extract the effective elastic properties such as the Young's modulus and Poisson's ratio. Preliminary results indicate that for low porosity samples $(\varphi=5-15 \%$ e.g. for consolidated material) the effective Young's modulus, averaged over the three spatial directions, conforms to the analytical approximation for random porous materials. For highly porous samples (up to $\varphi=50 \%$ e.g. loosely consolidated material from a chalk cliff), the results deviate from the analytical estimate, which could result from the limited subvolume and the weaker connectivity between the grains in the sample.

The findings of this study show that it is not only possible to derive meaningful values for the mechanical properties of nanoporous rocks using X-ray tomography and finite elements simulation but also provide a deeper understanding of the dependence of these properties on the micro- and nanostructure. Therefore, studies such as this can supplement results from macroscale testing to help engineers and scientists to assess the risk of erosion in chalk cliffs, as well as risks associated with drilling and production of oil and gas in chalk hydrocarbon reservoirs [5]. 
[1] T. Weitkamp et al., Developments in X-ray Tomography II of Proceedings SPIE (1999), p. 311-317

[2] D. Müter et al., Computer \& Geosciences 49 (2012), p. 131-139

[3] NETGEN meshing software, J. Schöberl, Vienna Institute of Technology

[4] FEBio, Musculoskeletal Research Laboratories, University of Utah

[5] The authors thank Finn Engstrøm for providing example chalk samples. Funding was provided by Mærsk Oil and Gas A/S and the Danish National Advanced Technology Foundation ( $\mathrm{P}^{3}$ Project), the Danish Council for Independent Research (via DANSCATT) and the European Commission (FP7 Fellowship TOMOMECH and the ITN Grant MINSC).

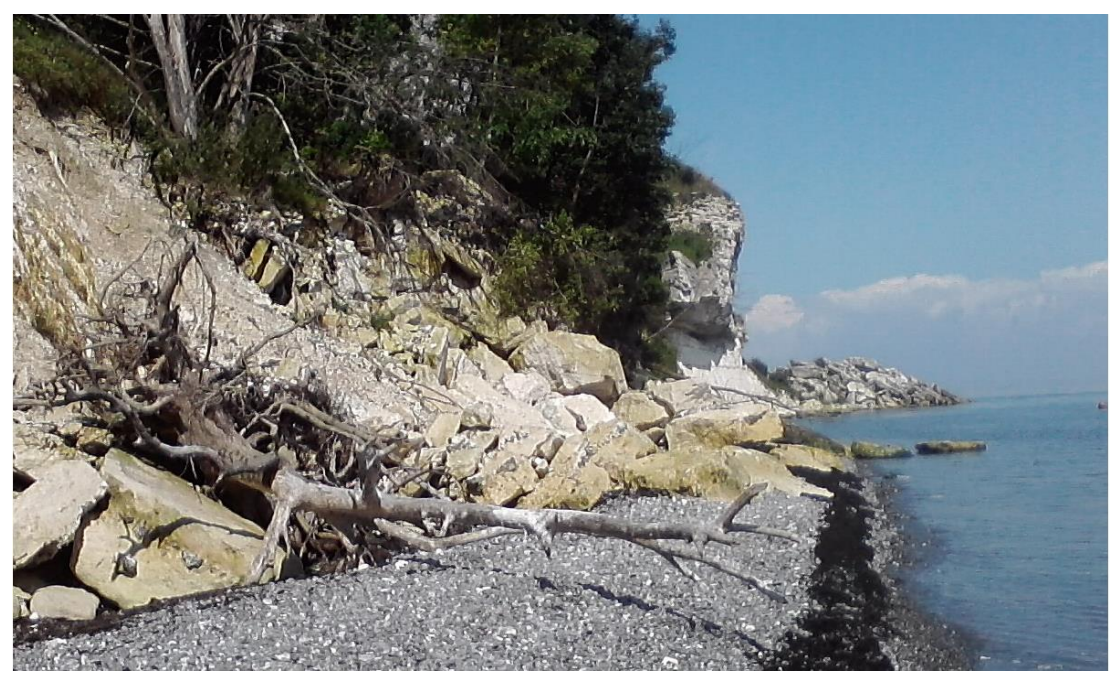

Figure 1. Chalk cliff at Stevns Klint, Denmark showing partial collapse of the cliff

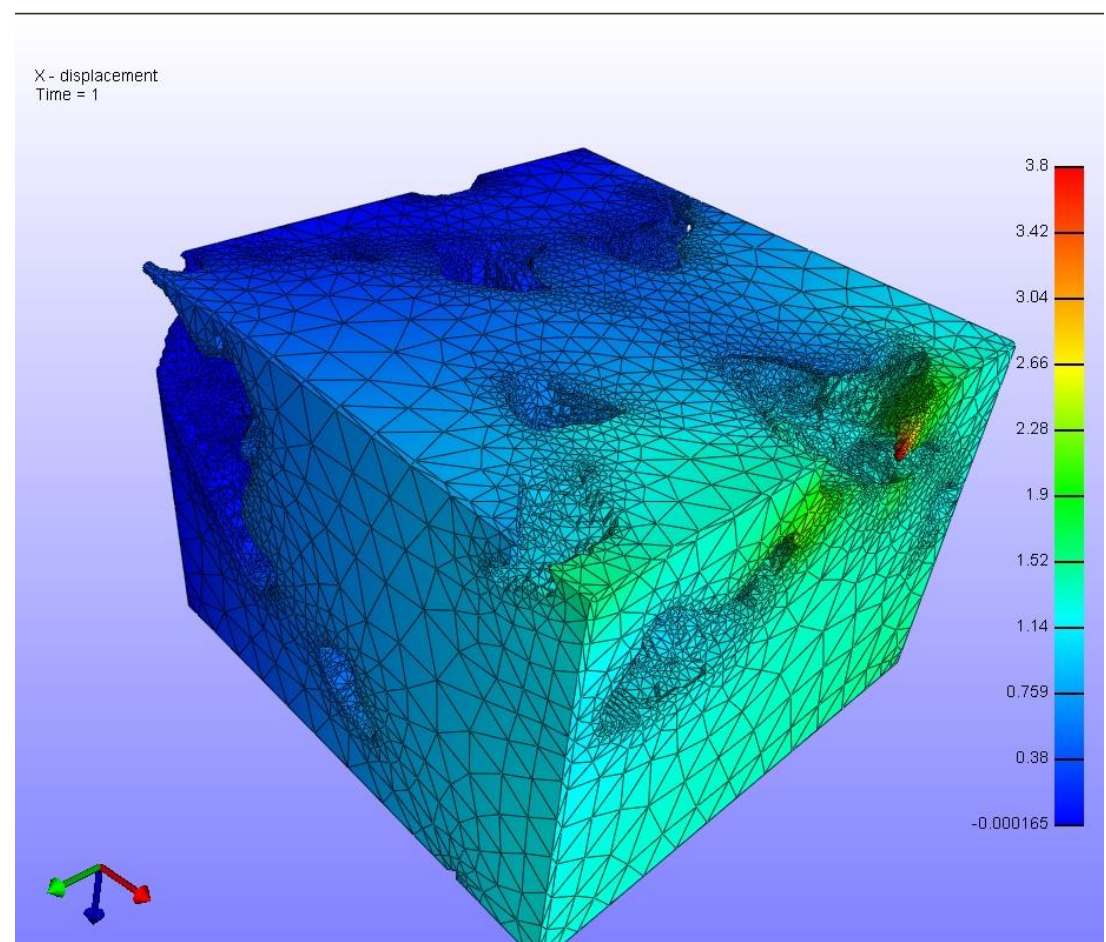

Figure 2. Meshed subvolume ( $3.4 \times 3.4 \times 2.5 \mu \mathrm{m})$ of a low porosity chalk sample; the sample is under tension along the $x$-axis (red axis), i.e. the back side of the cube is fixed while a tensile load is applied to the front. The color code indicates the resulting displacement along the the $x$-axis. 